\title{
BMJ Open Hospital postdischarge intervention trialled with family caregivers of older people in Western Australia: potential translation into practice
}

\author{
Samar M Aoun, ${ }^{1,2}$ Roswitha Stegmann, ${ }^{3}$ Susan Slatyer, ${ }^{3,4}$ Keith D Hill, ${ }^{5}$ \\ Richard Parsons, ${ }^{6}$ Rachael Moorin, ${ }^{7,8}$ Mary Bronson, ${ }^{9}$ Debbie Walsh, ${ }^{10}$ \\ Christine Toye $\mathrm{e}^{3,4}$
}

To cite: Aoun SM, Stegmann R, Slatyer S, et al. Hospital postdischarge intervention trialled with family caregivers of older people in Western Australia: potential translation into practice. BMJ Open 2018;8:e022747. doi:10.1136/ bmjopen-2018-022747

\section{- Prepublication history for} this paper is available online. To view these files please visit the journal online (http://dx.doi. org/10.1136/bmjopen-2018022747).

Received 25 March 2018 Revised 26 July 2018 Accepted 28 September 2018

Check for updates

(C) Author(s) (or their employer(s)) 2018. Re-use permitted under CC BY-NC. No commercial re-use. See rights and permissions. Published by BMJ.

For numbered affiliations see end of article.

Correspondence to Professor Samar M Aoun; s.aoun@latrobe.edu.au
There is lack of a suitable assessment tool that can be used routinely and systematically by hospital staff to address family caregivers' (FCs') support needs. This paper describes a novel approach to identifying and addressing FCs' needs following hospital discharge of the older person receiving care.

Setting and participants FC recruitment occurred on the patient's discharge from a tertiary hospital in Western Australia; 64 completed the study; $80 \%$ were female; mean age 63.2 years.

Intervention The Further Enabling Care at Home (FECH) programme was delivered over the telephone by a specially trained nurse and included: support to facilitate understanding of the patient's discharge letter; caregiver support needs assessment and prioritisation of urgent needs; and collaborative guidance, from the nurse, regarding accessing supports.

Results Sixty-four FCs completed the FECH programme. The top three support needs identified by the FCs were: knowing what to expect in the future $(52 \%)$, knowing who to contact if they were concerned (52\%) and practical help in the home (36\%). The telephone-based outreach service worked well and was convenient for the nurse and the FCs, and saved on transport, time and money. Most of the FCs appreciated the systematic approach to identify and articulate their needs and were satisfied with the support they received, mainly navigation through the systems, problem solving, self-care strategies, explanation of illness, symptoms and medication and access to after-hours services.

Conclusions In order to guide services which may consider adopting this systematic approach to supporting FCs and integrating it into their routine practice, this evaluation of the $\mathrm{FECH}$ programme has described the processes implemented and highlighted the factors that hindered or facilitated these processes to engage caregivers with appropriate services in a timely manner. Positive feedback indicated that the programme was a useful addition to hospital discharge planning.

Trial registration number ACTRN12614001174673; Results.
Strengths and limitations of this study

- This paper describes a novel programme implemented to support home caregiving for older people discharged from hospital.

- Provides indications of the support priorities of family caregivers to improve service provision.

- Identifies barriers to, and facilitators of, family caregivers' engagement with supportive resources and services.

- Reports programme implementation in one particular setting.

- Future research could evaluate efficacy and implementation in other healthcare settings (real-world practice).

\section{BACKGROUND}

For older people receiving care and in poor health, readmissions to hospital are potentially distressing for them and costly for the healthcare system, yet sometimes these can be avoided with well implemented discharge planning. ${ }^{1}$ A key component of effective discharge planning is to closely involve the family caregivers. ${ }^{2}$ However, in a recent study investigating returns to hospital after discharge from a short stay ( $<72$ hour) Medical Assessment Unit (MAU) in an Australian tertiary hospital, communication issues at the time of discharge presented a significant challenge. ${ }^{3}$ There was limited opportunity for the staff to liaise with family caregivers in the time available prior to discharge. In particular, it was evident that there was insufficient time for the staff to consider caregivers' needs and communicate plans to address likely future health crises for patients. ${ }^{3}$ This concern is compounded by the lack of a suitable assessment tool for caregivers' needs that can be used routinely and systematically by hospital staff in this field. 
The Carer Support Needs Assessment Tool (CSNAT) is one such evidence-based validated tool that has been used successfully to assess caregivers' needs in community palliative care in a systematic manner. In a trial conducted in palliative home care, it was effective in reducing caregiver strain, ${ }^{4}$ and was considered relevant, beneficial and easy to use by caregivers and nurses ${ }^{56}$ who recommended including it in the service's routine practice. The tool is a supportive family caregiver intervention and its use (the CSNAT Approach) is caregiver-led but facilitated by the healthcare professional. It adopts a screening format, structured around 14 broad support domains which fall into two distinct groupings: those that enable the caregiver to care; and those that enable more direct support for caregivers. ${ }^{7}$

The Further Enabling Care at Home (FECH) programme incorporated the CSNAT approach and was trialled as an outreach support programme for family caregivers of older people discharged from the MAU in a randomised controlled trial (RCT). The programme was shown to be effective in improving the caregiver's preparedness to care and decreased caregiver strain and distress. ${ }^{89}$ However, whereas in earlier work, the patient's usual nurse embedded implementation of the CSNAT in their practice, ${ }^{6}$ the $\mathrm{FECH}$ programme was delivered by a dedicated nurse as an outreach programme following hospital discharge. The FECH nurse was specially trained and was additional to the care team. This change was necessary because of the rapid discharge process implemented in the MAU which placed significant constraints on the time of the existing staff.

This article describes the details of the implementation of the FECH programme, as documented by the FECH nurse, including: adherence to, or deviation from, planned processes; information provided to caregivers and the resources to which they were referred; the extent to which caregivers engaged with resources; the contextual factors that were barriers to or facilitators of access and engagement; and time taken to implement processes. This information will serve as guidance to other services which may contemplate implementing such a programme.

\section{Objectives}

In the context of the successful implementation of the FECH project, the objectives of this article are to:

- Describe the implementation processes for this dedicated nurse role interfacing with the caregivers of older patients discharged home from the MAU.

- Highlight the barriers and facilitators of the implementation of the programme.

- Assess the utility of the CSNAT in this setting.

- Assess the feasibility to translate the programme into regular clinical practice.

\section{METHODS}

\section{Setting and participants}

Recruitment occurred on the patient's discharge from the MAU which was a short stay 36-bed assessment unit providing treatment for acutely ill complex medical patients. Most patients admitted to the MAU are aged $\geq 65$ years and remain for up to 72 hours on this unit, prior to discharge, or transfer to another inpatient team. ${ }^{8}$

Eligible dyads were patients aged 70 years or older admitted to MAU during the recruitment period (April to November 2015) and discharged directly home, and their adult family caregivers. The family caregivers needed to speak, read and understand English. A family caregiver was defined as a family member or friend who provided unpaid personal care, support and assistance to the patient. ${ }^{10}$

\section{Patient and public involvement}

This study was informed by previous work in which patients readmitted to the unit within 28 days of discharge, their family caregivers and the staff were interviewed about their experiences. ${ }^{3}$ Findings revealed a need for a programme such as that tested in the study reported here, in particular to help prepare caregivers to continue in their caregiving role, and the need for related outcome measures also became apparent from those findings. Patients were not involved in the RCT design but caregivers were included in the reference group for the study which helped to inform study processes. Patients and their caregivers were offered an opportunity to contribute qualitative data about their experiences of the intervention when caregivers had received this. All study participants were advised that they could request a summary of study findings which were also presented at carer conferences, in which caregivers were included as delegates.

\section{Description of the FECH nurse role and the intervention}

The FECH nurse implemented the programme through a number of preplanned telephone contacts with family caregivers following the patient's discharge from hospital. The research protocol designated three contacts. There was to be an initial contact (contact 1) within 1 week of the discharge (after informed consent to participation and baseline measures were obtained), in which the FECH nurse introduced herself and the programme, and then scheduled a second contact within 7-10 days of the discharge, at a time convenient to the caregiver. Contact 2 was planned to (a) determine and respond to the extent to which the family caregiver understood the copy of the discharge letter from the hospital MAU physician to the general practitioner (GP) that was provided as part of routine care; (b) administer the CSNAT to the family caregiver, resulting in the caregiver's self-identified and prioritised support needs; and (c) guide the caregiver to engage with existing resources to address the three highest prioritised needs identified from the CSNAT and the discharge letter review. Contact 3 , the final telephone contact, was designated to occur a few days later (within 2 weeks of the discharge) to determine if access to supportive resources had been achieved as planned and providing further advice if appropriate. Figure 1 shows the three FECH contacts occurring between collection of 


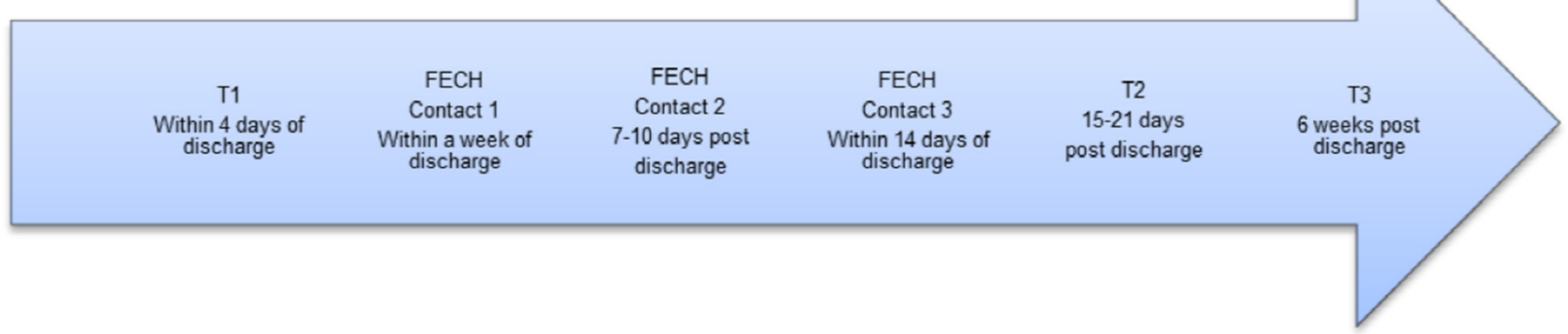

Figure 1 Planned FECH contacts and intervention points. FECH, Further Enabling Care at Home; T1, baseline; T2, follow-up.

baseline (T1) and follow-up (T2) outcome measures for those in the trial's intervention group.

Training for the FECH nurse included two components. The first component comprised the principles of the approach to be used and the administration of the tool which were presented within the context of palliative care in a toolkit developed by Austin et al. ${ }^{11}$ The second component was the use of a previously developed resource manual that helped to guide access to supports for caregivers and assist with family caregivers' linkage and engagement with appropriate existing resources.

\section{Data collection}

In order to facilitate a process evaluation, the FECH nurse collected data during programme implementation. These data comprised the demographic characteristics of the care recipients and the family caregivers, the frequency and length of the FECH contacts with the caregivers, information on the discharge summary, the CSNAT administration, the support needs and actions taken to meet the top three needs and caregivers' reports of issues encountered when engaging with services and resources. Descriptive and reflective field notes of the conversations with each caregiver were taken during and straight after the phone calls. Outcome measures at T1, T2 and T3 were collected by the research assistant as described previously. ${ }^{9}$

\section{Analyses}

The quantitative data collected by the FECH nurse were analysed through Excel Analysis ToolPak. Descriptive analyses were undertaken to provide a profile of participants and other process indicators. Analysis of CSNAT data was undertaken using IBM SPSS V.22 statistical software.

The field notes were transcribed and organised to reflect the content of FECH nurse-caregiver discussions at each contact. For example, field notes from contact 2 were summarised to describe the caregivers' overall understanding of patients' conditions; responses to the discharge summary, and the FECH nurse guidance given to address caregivers' prioritised needs. Similarly, field notes documenting contact 3 captured caregivers' level of engagement in the suggested supportive resources.

\section{RESULTS}

\section{Participants}

In total, 77 of the 163 caregivers who provided baseline measurement data were allocated to the intervention ${ }^{9}$ and $64(83.1 \%)$ completed the FECH programme. Seventeen per cent $(n=13)$ of the caregivers did not complete the three contacts because the patient was very ill or the caregiver felt too stressed or too busy $(\mathrm{n}=10)$, or the caregiver did not respond to the FECH nurse contact attempts and was lost to follow-up $(n=3)$.

Table 1 presents the characteristics of the 64 family caregivers who completed the intervention and the characteristics of the patients for whom they provided care. Eighty per cent of the caregivers $(n=51)$ were female. Daughters and wives represented the majority of family caregivers $(\mathrm{n}=44,68.8 \%)$. The age of the caregivers ranged from 25 to 89 years old, with the mean age 63.2 years (SD 12.8). The age of the patients ranged from 70 to 99 years old, with a mean age of 84.6 years (SD 6.7).

\section{FECH contacts with family caregivers}

Table 2 summarises the post discharge time and duration of phone calls for the three contacts. Sixty-three per cent of family caregivers were available for the arranged contact 2 on the first attempt and $77 \%$ of family caregivers responded to contact 3 on the first attempt. One caregiver needed seven attempts to be recontacted and $28 \%$ and $23 \%$ of second and third contacts, respectively, occurred outside business hours. Contact 1 was the shortest (mean $15.4 \mathrm{~min}$ ) as it was an introduction to the programme. Contact 2 was the longest as expected (mean $59.7 \mathrm{~min}$ ). This contact covered discussion about the discharge summary, administration of the CSNAT, and linking the family caregiver to appropriate existing resources. Contact 3, the evaluation and follow-up contact, was frequently a shorter contact with a mean of $28.3 \mathrm{~min}$.

Although the contact points were based on the team's knowledge of caregivers' needs and the clinical expertise of hospital-based researchers about the short-stay setting and immediate postdischarge context, there was a change to the timing of the nurse contacts from those planned and presented in figure 1 . Contact 1 was implemented 
Table 1 Characteristics of family caregivers and patients who completed the intervention $(n=64)$

\section{n (\%)}

\begin{tabular}{ll}
\hline Family caregiver & \\
Gender & \\
Female & $13(79.7)$ \\
Male & \\
Age & $63.2(12.8)$ \\
Mean (SD) & $63.5(25,89)$ \\
Median (range min., max.) & \\
Relationship & $23(35.9)$ \\
Daughter & $21(32.8)$ \\
Wife & $11(17.2)$ \\
Son & $1(1.6)$ \\
Husband & $8(12.5)$ \\
Other & \\
Living with patient & $28(43.6)$ \\
No & $36(56.4)$ \\
Yes & \\
Patient & \\
Gender & $31(48.4)$ \\
Female & \\
Male & \\
Mean (SD) & \\
Median (range min., max.) & \\
\hline Me & \\
\hline
\end{tabular}

max., maximum; min., minimum.

within up to 9 (instead of 7) days of the discharge, contact 2 within 24 (instead of 10) days and contact 3 within 40 (instead of 14) days. These changes were required to fit in with the multiple, and often unpredictable, commitments of the family caregivers or their limited availability.

\section{Discharge summary}

Exploring family caregivers' understanding of discharge information, prior to the CSNAT assessment in contact
2, tended to elucidate their awareness of the patients' medical situation, what happened during the hospital stay and the follow-up instructions. Most participants (81.3\%) showed a good understanding of the medical situation and deemed the explanations received from the hospital staff or the GP to be sufficient.

Some of the caregivers' questions related to medical terms or the patient's symptoms and were addressed by the FECH nurse. When further clarification was needed, the FECH nurse directed the caregiver to their GP or the relevant physician if a follow-up outpatient appointment had been arranged. During these discussions, some caregivers raised issues that had confused them which included: surprise at the early discharge, confusion about the health professionals involved in the hospital care of their relative, the management of follow-up appointments and further investigations and the medical diagnosis. Caregivers also expressed apprehension if underlying reasons for the patient's presenting symptoms had not been found and requested guidance about managing symptoms if these reappeared.

\section{CSNAT administration: support needs and actions addressing them}

The CSNAT form was posted or emailed to the caregiver after contact 1 . Accessing this form prior to contact 2 granted the caregiver time to consider the different domains; reinforced the value of a discussion with friends, relatives and the patient as appropriate; and empowered the caregiver to determine what they wanted to disclose and whether this was the right time for them. During contact 2, discussing the domains opened up the conversation with some caregivers. Others focused just on the domains where they felt that they needed more support. The aim was to establish their three main concerns and to implement appropriate support. As presented in figure 2, the overall top three support needs identified by the family caregivers were: knowing what to expect in the future (52\%), knowing who to contact if concerned $(52 \%)$ and practical help in the home (36\%). The 'other' category consisted of a caregiver's concern about their mother's driving or about organising social activities for their mother. The direct support needs of the caregivers,

Table 2 Duration of phone call and time to contact post discharge

$\begin{array}{lll}\text { Contact } 1 & \text { Contact } 2 & \text { Contact } 3 \\ n=64 & n=64 & n=52\end{array}$

Duration of call (min)

$\begin{array}{lllr}\text { Mean (SD) } & 15.4(9.6) & 59.7(24.1) & 28.3(17.7) \\ \text { Median (range) } & 12.0(5-46) & 59.5(15-120) & 23.5(5-90)\end{array}$

Contact postdischarge (days)

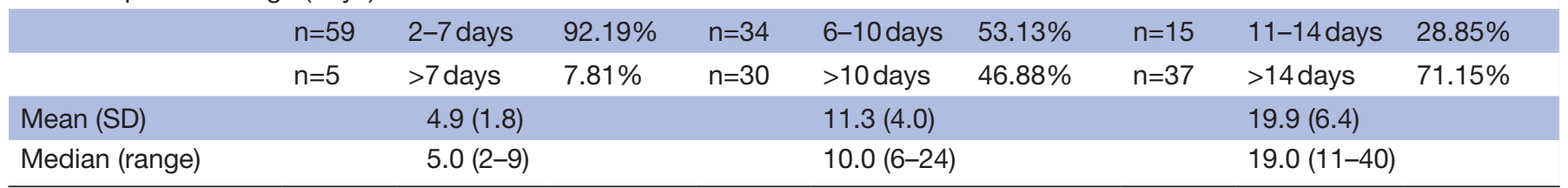


...knowing what to expect in the future

...knowing who to contact if you are concerned

...practical help in the home

...having time for yourself in the day

...your financial, legal, or work issues

...managing your relative's symptoms

...understanding your relative's illness

...looking after your own health

...talking with your relative about his or her illness

...equipment to help care for your relative

...dealing with your feelings and worries

...getting a break from caring overnight

...providing personal care for your relative

...your beliefs or spiritual concerns

.... Other

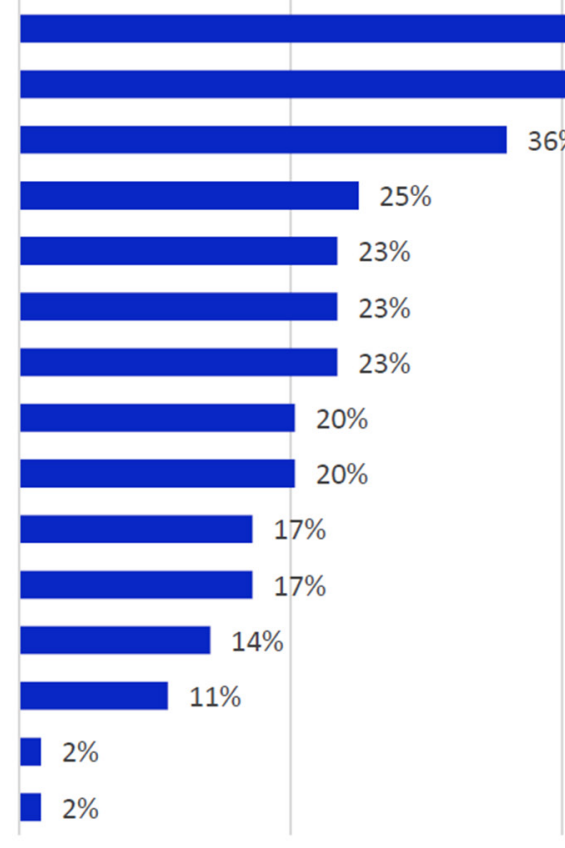

$52 \%$

$52 \%$

0

20

40

60

Figure 2 Percentage of family caregivers expressing need for more support within each Carer Support Needs Assessment Tool domain.

as opposed to the enabling needs to help them to care, featured in the top support needs, namely practical help in the home, having time for themselves in the day and financial, legal and work issues.

Table 3 summarises the nurse actions that were implemented to address the caregivers' needs in the top three priority areas. Overall, actions were centred around dealing with the illness and symptoms, home care and residential care, the legal aspects and self-care strategies.

\section{Engagement of caregivers with resources and services}

A number of issues influenced whether support was achieved. Figure 3 summarises the issues reported, during contact 3 , by caregivers that influenced their engagement with resources and services.

During discussion with caregivers, the FECH nurse identified barriers to engaging with resources and services, comprising: (a) limited caregiver time; (b) a shift in priorities; (c) patient factors (their decision and reluctance to access services, deterioration soon after discharge); (d) dealing with a new diagnosis and unexplained symptoms, and confusion that could arise when different services were involved; (e) the caregiver's role and situation; (f) limitations of services and $(\mathrm{g})$ financial aspects.

\section{Supported the family carer's request to increase existing support and to ask for help}

In contrast, perceived facilitators included: (a) the caregiver time availability, (b) the caregiver's engagement and good relationship with the patient, (c) the caregiver's own medical experiences or background knowledge, (d) the patient's compliance, (e) existing services (including family and GP support) and (f) caregiver empowering approach.

Most of the support services discussed during the CSNAT conversation related to navigation through the system (health and aged care), problem solving, self-care strategies, explanation of illness, symptoms and medication, and access to services such as after-hours GP service, social worker and aged care services.

Caregivers indicated whether the support identified in the shared care plan had been achieved by the time of the contact 3 discussion:

- $9.4 \%$ felt the support exceeded what they had expected.

- $7.8 \%$ felt that the support was fully achieved as planned.

- 50\% reported that their support was partially achieved. Often caregivers' priorities shifted when the patient's condition improved or awaiting treatment decisions, support became less urgent, and therefore contact with services was not arranged or postponed.

- $4.7 \%$ had no support achieved because in the timeframe of the study, patients and caregivers circumstances changed.

- $28.1 \%$ did not undertake an evaluation as no priority support needs were identified and by consequence no action plan was warranted.

The field notes captured caregivers' comments provided during the final conversation with the FECH nurse which suggested that the programme was a useful addition to hospital discharge planning. For example, one caregiver had reported to the FECH nurse that: 
Table 3 Summary of nurse actions to address the top three family carers' support needs

\section{Knowing what to expect in the future when caring for your relative}

IIIness and symptoms Explored carer's understanding of the patient's illness, current situation, symptom control.

Discussed, for example, diabetes, Parkinson's disease, dementia, renal failure, palliative care.

Suggested attend doctors' appointments with patient or, with patient's consent, speak to doctor.

Advised on doctors' referral options to specialists and allied health professionals.

Verified questions carers may like to ask the doctor and discussed new strategies.

Reinforced plan to organise and attend family meeting with the doctor.

Home care and

residential care

Discussed available services (eligibility criteria and assessment process).

Referred to hospital social worker as indicated.

Recommended useful aged care services to enable carers' informed decisions about home care services, residential care questions and cost calculations.

Reinforced the family carer's follow-up with previous or current aged care assessments.

Referred to assessment services to arrange domestic support.

Navigated family carers through the options and listened to their concerns.

Legal aspects Discussed legal considerations if mental or physical health of relative declines.

Recommended to contact appropriate services for more information and forms.

\section{Knowing who to contact if you are concerned about your relative}

Discussed or confirmed appropriate actions with family carer in a medical emergency-focus on after-hours services.

Used case scenarios to demonstrate various situations and responses.

Suggested implementing a contact list of useful services.

Discussed strategies to stay calm in a stressful situation.

Reinforced the need to seek help early before a situation escalates.

Explained emergency respite, personal alarms, other equipment resources and continence services.

Discussed the family carer's awareness of legal decisions made by the patient for the future.

\section{Practical help in the home}

Discussed domestic help options and initiated referrals to appropriate agencies.

Explored existing support (family members, other support services).

...after discharge from the hospital you don't get the follow-up ...have an understanding now ...can see where to go from here...I did not pick it up from the book.

Other expressions of satisfaction, as reported in the nurse's field notes by several caregivers, included:

...don't feel so up in the air anymore.

...extremely helpful ...explaining a lot, would have been floundering otherwise.

...everything seems to be falling into place.

...the best thing about your call, it made me think, you normally just do and don't think about what I really need and what I need most.

...really, really helpful, I was not aware about the services.

...good questions, make me think....great opportunity ...nice to reflect, to know to have someone with the knowledge ...I can tap in.

\section{DISCUSSION}

To our knowledge, this is the first implementation of the CSNAT for family caregivers of older adults as part of an outreach programme following discharge from acute hospital care and administered by a dedicated nursing role. The usefulness of this programme is considered, as follows, from the perspective of the caregivers and the FECH nurse who experienced implementation of the programme.

This process evaluation indicated that the FECH programme had a number of strengths:

- It provided family caregivers with the equivalent of a one stop shop where their most pressing needs, whether for information or support, were either addressed or channelled to appropriate services.

- The FECH nurse's knowledge of existing resources (supported by the FECH manual) could guide caregivers to 'navigate' the maze of available services and access appropriate and timely support.

- It provided access to telephone-based nursing expertise at a time when caregivers were dealing with their 

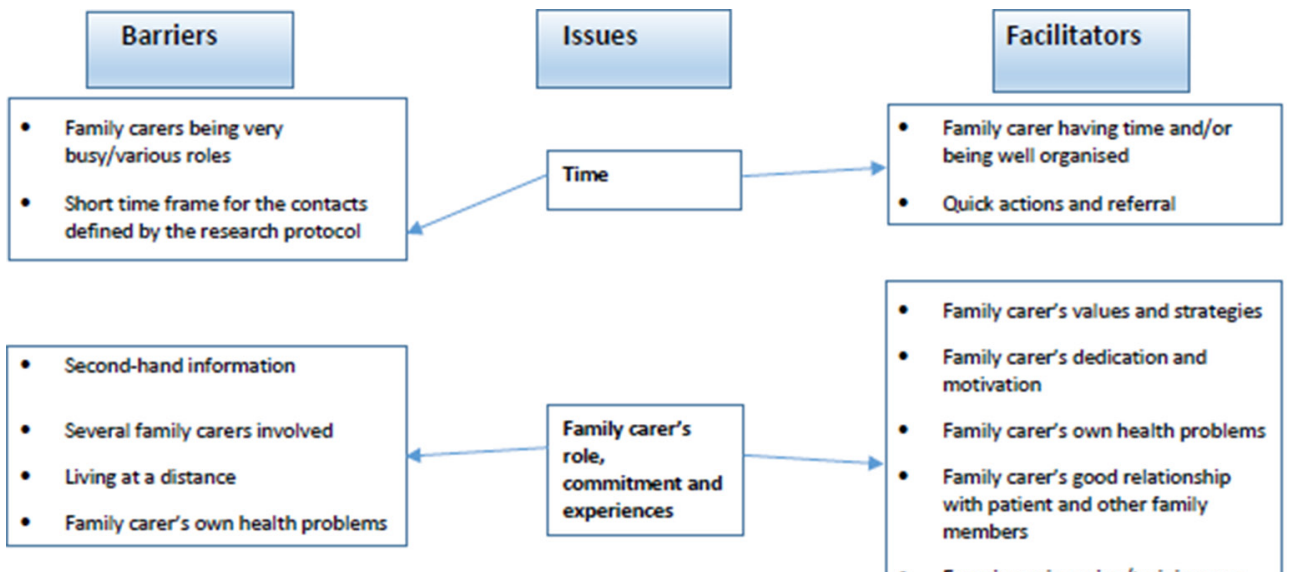

- Family carer's values and strategies

- Family carer's dedication and motivation

- Family carer's own health problems

Family carer's good relationship with patient and other family members

- Experience in caring/training as a health professional and knowing

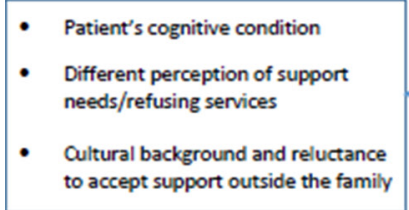
services
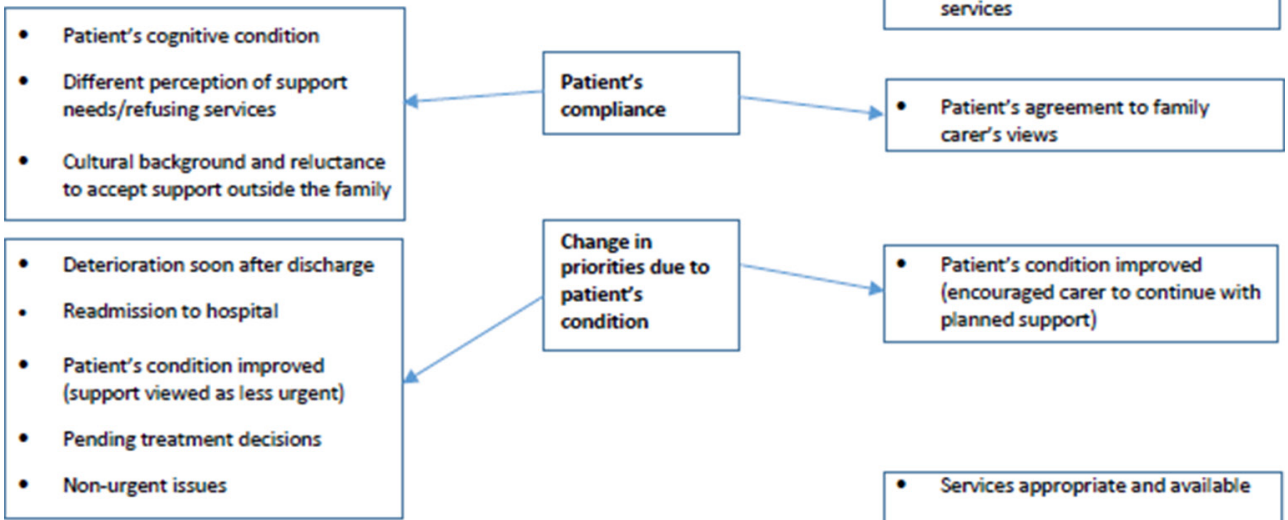

- Limitation and lack of services
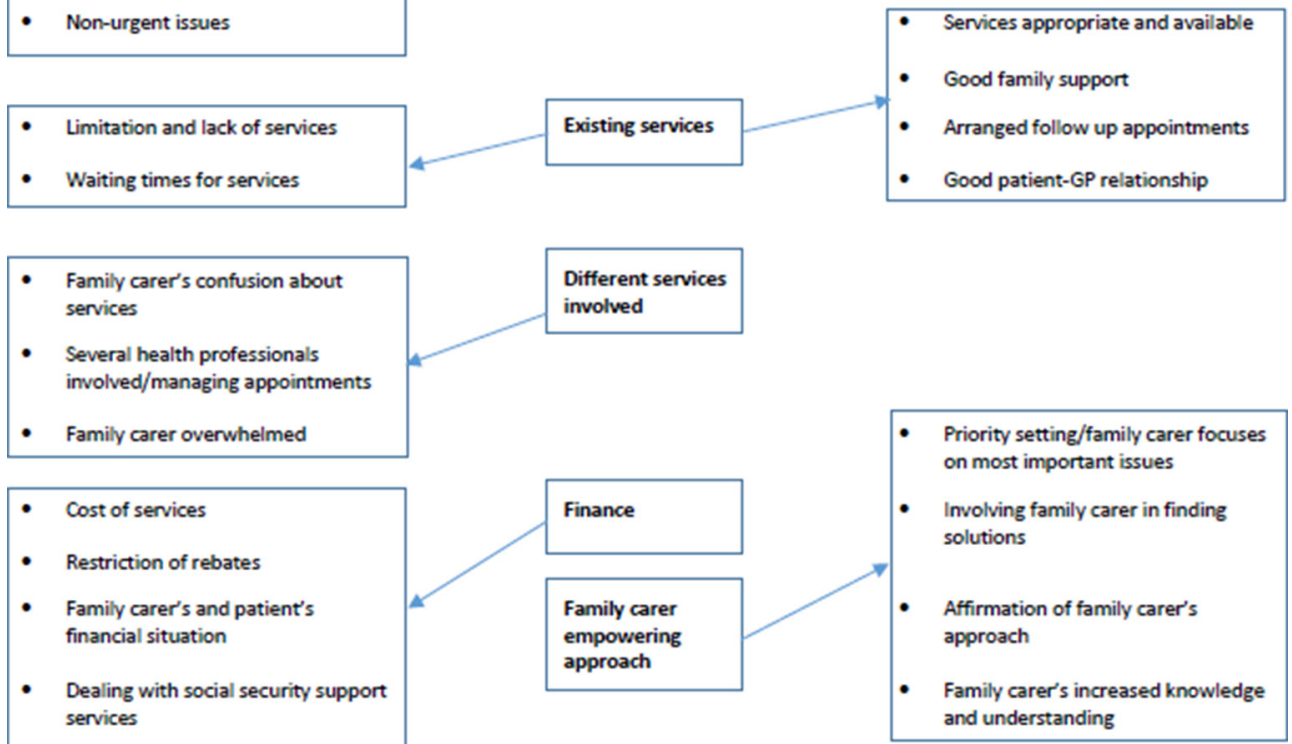

Figure 3 Factors related to engagement of family carer with resources and services: barriers and facilitators.

relative's and their own needs, perhaps confused and under stress.

- It provided a systematic approach to identifying and addressing caregivers' needs.

- It provided a caregiver-led process that crystallised caregivers' most pressing needs that were then feasible to articulate.

- The discussion around the discharge letter helped clarify what was needed in the first instance.

The caregivers' satisfaction was reflected in the significant positive results of the trial where, compared with the control group, the intervention group were better prepared to care and experienced less strain and distress. ${ }^{9}$

The positive outcomes of the FECH programme mirrored those achieved in community palliative care ${ }^{4}$ which was the original setting in which the CSNAT was developed. ${ }^{7}$ For this hospital setting, most CSNAT domains were well used, reflecting their relevance to this setting. Whereas the top need 'knowing what to expect in the future' was similar between the two settings, 'dealing with your feelings and worries' featured low in this hospital setting while it was the third most stated 
need in the palliative care setting. This may be because the looming death in the palliative care setting generates more need for such personal support. It was also reassuring to note that, from the FECH nurse's perspective, the CSNAT gave caregivers the opportunity to voice their concerns about their own direct needs and not just those they need to provide care. From earlier experience, it is envisaged that caregivers gained benefit from the recognition accorded to their needs as visible, legitimised and acknowledged. $^{4}$

However, there were a couple of stated needs that did not quite fit in the domains or were difficult to separate: caregivers did not see their need for a longer holiday or a short break of a few days necessarily covered in the domain 'getting a break from caring overnight'. This may be different in the palliative care situation, where the caregiver would not want to leave the patient for a longer timeframe. Also, the domain 'knowing what to expect in the future' was interpreted differently by some caregivers as their coping strategy emphasised 'each day as it comes'. This domain inter-related with other domains such as understanding their relative's illness, symptom control and practical help. A larger trial with more sites would be needed to ascertain whether additions or changes to the current CSNAT domains are warranted, including discussion with the tool developers.

The telephone-based outreach service worked well and was convenient for the nurse. During a telephone conversation, the electronic FECH resource manual and appropriate websites could be quickly scanned for information that was provided immediately, or compiled after the phone call and either emailed or posted by the nurse. The caregiver did not depend on any transport for the appointment, and this was flexible and saved time and money. There is a growing evidence that programmes for caregivers delivered over the telephone can be successful. $^{12} 13$

The FECH nurse in this research project demonstrated the required characteristics for the successful implementation of the programme: The nurse had acute care knowledge relevant to the care of older people in poor health, knowledge of how to access local services, understanding of the family caregiver role, the capacity to work flexible hours to fit in with caregivers' needs and the skills to support the caregiver during the process of reflection and self-assessment. This position was not embedded within the hospital during the trial because of the time constraints on existing staff in a setting with a short hospital stay ( $<72$ hours), and it included elements of process documentation that might be greater than those required in practice. For the purpose of this time-limited research project, only one contact involving the CSNAT was possible (contact 2), while the preceding contact was for planning and the following one for evaluation. However, in routine clinical practice, service providers would need to make the judgement when to introduce the CSNAT and how often to include it in the caregiving journey.
From the organisational perspective, the hospital has supported this trial and recognises the value of the FECH programme as it aligns with the organisation's commitment to National Safety and Quality Health Service Standard 2-Partnering with Consumers. ${ }^{14}$

\section{Limitations}

This study's limitations include the small sample size, the brief follow-up period, the programme being undertaken with just one service and in one geographical location and the exclusion of caregivers with limited English ability to speak and write. It is also likely that the caregivers in most need of support may not have been able to participate because of their time limitations or stress levels due to their care recipients having higher physical and cognitive support needs. Although there were heavy demands on caregivers' time when providing data at three time points plus receiving the intervention in three sessions, the attrition rate was reasonable at $19.5 \%$ as reported in ref 9 . We did not undertake a comparison between participants and non-participants as no data were collected on those who declined to participate when approached at the hospital. The caregivers' brief feedback reported in this manuscript is based on the nurse's field notes. The qualitative feedback from the in-depth interviews with the family caregivers will be reported in detail in a forthcoming publication.

\section{CONCLUSIONS}

This process evaluation has described the systematic and caregiver-led FECH programme, a phone-based nursing outreach service delivered immediately postdischarge from an acute medical unit. Caregivers' responses in terms of their identified needs for support, and apparent barriers and facilitators to caregiver engagement with supportive resources are reported in an attempt to provide detailed information to services who may consider adopting this systematic approach to supporting family caregivers.

It is recommended that ongoing assessment to anticipate caregiver needs should be integrated into routine practice rather than be 'crisis' driven. ${ }^{15}$ However, the challenges that need to be tackled for integration into practice include: developing an intervention relevant to caregivers themselves and that fits into practice; testing the effect of the intervention; and changing practitioner behaviour. This programme proved successful as several of these challenges have been met. Given the positive outcomes, what needs to be established is whether organisations are willing to integrate the FECH programme into practice. Adopting and sustaining an intervention for family caregivers within an organisation depends on the relevance of the intervention to the organisation's identified goals and priorities, available resources and existing strategies. ${ }^{16}$

Author affiliations

${ }^{1}$ School of Psychology and Public Health, La Trobe University, Bundoora, Victoria, Australia 
${ }^{2}$ The Perron Institute for Neurological and translational Science, Perth, Western Australia, Australia

${ }^{3}$ School of Nursing, Midwifery and Paramedicine, Curtin University, Perth, Western Australia, Australia

${ }^{4}$ Centre for Nursing Research, Sir Charles Gairdner Hospital, Perth, Western Australia, Australia

${ }^{5}$ School of Physiotherapy and Exercise Science, Curtin University, Perth, Western Australia, Australia

${ }^{6}$ School of Pharmacy, Curtin University, Perth, Western Australia, Australia ${ }^{7}$ School of Public Health, Curtin University, Perth, Western Australia, Australia ${ }^{8}$ School of Population Health, The University of Western Australia, Perth, Western Australia, Australia

${ }^{9}$ Medical Division, Sir Charles Gairdner Hospital, Perth, Western Australia, Australia

${ }^{10}$ Department of Social Work, Sir Charles Gairdner Hospital, Perth, Western Australia, Australia

Acknowledgements In-kind support was provided by Sir Charles Gairdner Hospital's Centre for Nursing Research. The authors thank all the family caregivers for their involvement and participation in this study, and also hospital health professionals and community services for their willingness and readiness to share information and to assist with support. The authors also thank Rebecca OsseiranMoisson, Kathy Deas, Cathy Pienaar, Philippa Wharton and all members of the study's reference group for their assistance in this project. Associate Investigators from Sir Charles Gairdner Hospital not included as authors on this paper were: Sue Davis, Nurse Director Corporate Nursing Research \& Education; Dr Matthew Skinner, Consultant Physician, Medical Director, Medical Assessment Unit; Dr Sean Maher, Consultant Geriatrician and Head of the Department of Rehabilitation and Aged Care, and Dee Whitty, Clinical Nurse Specialist, Medical Assessment Unit.

Contributors SMA, SS, KDH, RP, RM, MB, DW and CT all made substantial contributions to the conception and design of this study. SMA led the development of this manuscript, with substantial input from RS. RS implemented the programme, also collecting the data included in this paper. SMA and RS analysed and interpreted the data. SS and CT helped guide data collection. All authors were involved in drafting the manuscript, or revising it critically for important intellectual content. All authors read and approved the final manuscript.

Funding This work was supported by a Department of Health of Western Australia SHRAC Research Translation Project grant; the School of Nursing, Midwifery and Paramedicine at Curtin University; and the School of Physiotherapy and Exercise Science at Curtin University.

Competing interests None declared.

Patient consent Not required.

Ethics approval Ethical approval for the study was obtained from the Human Research Ethics Committees of the Department of Health of Western Australia (2014-78), Curtin University (HR14-2015), and the participating hospital (2014-133).

Provenance and peer review Not commissioned; externally peer reviewed.

Data sharing statement It was a requirement of ethical approval for this study that the study data would not be transported off site either in electronic or hard copy format.

Open access This is an open access article distributed in accordance with the Creative Commons Attribution Non Commercial (CC BY-NC 4.0) license, which permits others to distribute, remix, adapt, build upon this work non-commercially, and license their derivative works on different terms, provided the original work is properly cited, appropriate credit is given, any changes made indicated, and the use is non-commercial. See: http://creativecommons.org/licenses/by-nc/4.0/.

\section{REFERENCES}

1. Fox MT, Persaud M, Maimets I, et al. Effectiveness of early discharge planning in acutely ill or injured hospitalized older adults: a systematic review and meta-analysis. BMC Geriatr 2013;13:70.

2. Bauer M, Fitzgerald L, Haesler E, et al. Hospital discharge planning for frail older people and their family. Are we delivering best practice? A review of the evidence. J Clin Nurs 2009;18:2539-46.

3. Slatyer S, Toye C, Popescu A, et al. Early re-presentation to hospital after discharge from an acute medical unit: perspectives of older patients, their family caregivers and health professionals. J Clin Nurs 2013;22:445-55.

4. Aoun SM, Grande G, Howting D, et al. The impact of the carer support needs assessment tool (CSNAT) in community palliative care using a stepped wedge cluster trial. PLoS One 2015;10:e0123012.

5. Aoun S, Deas K, Toye C, et al. Supporting family caregivers to identify their own needs in end-of-life care: qualitative findings from a stepped wedge cluster trial. Palliat Med 2015;29:508-17.

6. Aoun S, Toye C, Deas K, et al. Enabling a family caregiver-led assessment of support needs in home-based palliative care: potential translation into practice. Palliat Med 2015;29:929-38.

7. Ewing G, Brundle C, Payne S, et al. The Carer Support Needs Assessment Tool (CSNAT) for use in palliative and end-of-life care at home: a validation study. J Pain Symptom Manage 2013;46:395-405

8. Toye C, Moorin R, Slatyer S, et al. Protocol for a randomised controlled trial of an outreach support program for family carers of older people discharged from hospital. BMC Geriatr 2015;15:70.

9. Toye C, Parsons R, Slatyer S, et al. Outcomes for family carers of a nurse-delivered hospital discharge intervention for older people (the Further Enabling Care at Home Program): Single blind randomised controlled trial. Int J Nurs Stud 2016;64:32-41.

10. Aggar C, Ronaldson S, Cameron ID. Self-esteem in carers of frail older people: resentment predicts anxiety and depression. Aging Ment Health 2011;15:671-8.

11. Austin L, Ewing G, Diffin J, et al. The Carer Support Needs Assessment (CSNAT): a toolkit for practitioners: School of Health Sciences, University of Manchester or Centre for Family Research, University of Cambridge, 2015.

12. Bakas T, Austin JK, Habermann B, et al. Telephone assessment and skill-building kit for stroke caregivers: a randomized controlled clinical trial. Stroke 2015;46:3478-87.

13. Jackson $D$, Roberts $G, W u$ ML, et al. A systematic review of the effect of telephone, internet or combined support for carers of people living with Alzheimer's, vascular or mixed dementia in the community. Arch Gerontol Geriatr 2016;66:218-36.

14. Australian Commission on Safety and Quality in Health Care. National Safety and Quality Health Service Standards. Sydney: Australian Commission on Safety and Quality in Health Care, 2012.

15. Payne S, Morbey H. Supporting family carers: report on the evidence of how to work with and support family carers to inform the work of the Commission into the future of hospice care. Help the Hospices: UK, 2013:36.

16. Rycroft-Malone J, Harvey G, Seers K, et al. An exploration of the factors that influence the implementation of evidence into practice. J Clin Nurs 2004;13:913-24. 